\title{
Mándame cero besos. La sesión montessoriana
}

José Manuel VÁzquez-Romero

Universidad Pontificia Comillas vazquez@comillas.edu

DOI: https://doi.org/10.15366/bp.2020.24.025

Bajo Palabra. II Época. No 24. Pgs: 495-524

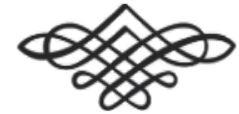


Recibido: 21/07/2020

Aprobado: $12 / 08 / 2020$

\section{Resumen}

El artículo analiza algunos pasajes del método montessoriano, concibiéndolo como si de una gestión del gozo (jouissance) infantil se tratara: la capitalización educativa de la compulsión a la repetición del nińo va derivando sucesivamente las pulsiones de éste hacia distintas casillas, desde la presentación del material educativo como objeto supuestamente predestinado del deseo, a la obsesión por la perfección y al histerismo en la inhibición como ejercicios didácticos en poco o nada concernidos por la funcionalidad del objeto, hasta llegar al escaque vacío de la falta constitutiva del sujeto-el ejercicio del cero.

Palabras clave: método Montessori, psicoanálisis lacaniano, pulsión, inhibición, cero, amor.

\section{Abstract}

The article analyses certain phases of the Montessori method, conceiving it in terms of infantile satisfaction (jouissan$c e$. Capitalising on this form of education, a child's compulsion and repetitiveness direct the drives of the child towards different areas which begins with the presentation of educational material as an object of desire and leads to the obsession for perfection, and to hysteria in inhibition as didactic exercises having little or no concern for the functionality of the object up to the point of producing a void gap or constitutive lack of the subject—-the zero exercise. 
Ein Nichts

waren wir, sind wir, werden war bleiben, blühend:

die Nichts-, die

Niemandrose.

(P. Celan, "Psalm”, Die Niemandrose, 1963)

Una nada

fuimos, somos, seremos siempre floreciendo: rosa de nada, de Nadie rosa.

(trad. de J. L. Reina Palazón)

Y todas las cosas para llegar a ser se miran en el vacío espejo de su nada

(Espacio)

(J. Á. VALENTE, Fragmentos de un libro futuro, 2000)

La psychoanalyse - mais ce n'est plus elle, elle n'est plus identifiable - oblige à penser une étrange élection de la dissociation: le choix d'un objet inobjectivable, qui s'incorpore et me divise - ou le choix de mon Moi par un sujet inidentifiable, qui m’incorpore et se divise

(Ph. Lacoue-Labarthe et J.-L. Nancy, La panique politique)

El hecho fundamental, il fatto fondamentale che mi condusse a determinare questo metodo, el método pedagógico, será la repetición, el placer de repetir:

Io stavo facendo le mie prime prove nell'applicare i principî e parte del materiale che mi ereano serviti molti anni prima all'educazione dei bambini deficienti, sopra i piccoli 
bambini normali di S. Lorenzo ${ }^{1}$, quando mi accadde di osservare una bambina di circa tre anni, che rimaneva profondamente assorta sopra un incastro solido, sfilando e infilando i cilindretti di legno nei loro posti rispettivi. L'espressione della bambina era di una sì intensa attenzione, che mi sembrò quella una manifestazione straordinaria: i bambini fino allora non avevano mai mostrato una tale fissità sopra un oggetto: e la mia convinzione sulla instabilità caratteristica dell'attenzione nel piccolo bambino, che passa senza posa da cosa a cosa, mi rendeva ancor più sensibile al fenomeno.

Io osservai intensamente la piccina senza disturbarla in principio e cominciai a contare quante volte ripeteva l'esercizio: ma poi, vedendo che continuava molto a lungo, presi la poltroncina su cui era seduta, e posi poltroncina e bambina sulla tavola; la piccolina raccolse in fretta il suo incastro, poi lo posò attraverso i braccioli della poltroncina, e mettendosi in grembo i cilindretti, continuò il suo lavoro. Allora invitai tutti i bambini a cantare: essi cantarono, ma la bambina continuò imperturbata a ripetere il suo esercizio anche dopo che il breve canto fu cessato. Io avevo contato quarantaquattro esercizi; e quando finalmente cessò, cessò in modo affatto indipendente dagli stimoli dell'ambiente che potevano disturbarla, quasi svegliandosi da un sonno riposante.

La mia impressione indimenticabile credo che somigliasse a quella provata da chi ha fatto una scoperta ${ }^{2}$.

(La bambina que inserta en y retira el cilindro de su horma, infila e sfila, como una posesa, una y otra vez, hasta cuarenta y cuatro, como una máquina, hasta que se fija, se apacigua.)

Meter y sacar, allá y acá, dentro y fuera, ausente y presente, fort-da. La compulsión a la repetición ansía suturar la brecha y cerrar el círculo que la ausencia del objeto del deseo abre (¿la mammella?), marrado desde el entonces del des-

\footnotetext{
1 "Le mie prime esperienze sui bambini normali furono iniziate nella prima metà del noviembre 1907.[/] Nelle due "Case dei Bambini» di San Lorenzo, avevo dal 6 gennaio nell'una e dal 7 marzo nell'altra, data della rispettiva inagurazione, applicato solo i giuochi della vita pratica e dell'educazione dei sensi, fino a tutto il luglio, epoca in cui un mese di vacanza avrebbe interrotto le lezioni" (M. Montessori, Il Metodo della Pedagogía Scientifica applicato all'educazione infantile nelle Case dei Bambini. Edizione critica, Roma: Edizioni Nazionale Montessori 2000, pp. 516 s.; en lo sucesivo, Il Metodo. Cfr., también, pp. 129ss.).

${ }^{2}$ M. Montessori, L'autoeducazione nelle scuole elementari, Milano: Garzanti 2000, pp. 61s. La niña normal de esa escena fundacional del método relevaría a la nińa deficiente, en la que se habría ensayado y perfeccionado el material y el método (cfr. Il Metodo, pp. 110ss.). El idiota sería sordo a la llamada del material educativo, teniendo que tomar la maestra la voz cantante: "... e credo che non il materiale didattico, ma questa mia voce che li chiamava, destò i fanciulli, e li spinse ad usare il materiale didattico e a educarsi” (id., p. 120). Pero, a pesar de los óptimos resultados de esa educación, la maestra remata afónica y exangüe: "ma debbo confessare che [...] una especie de esaurimento mi postrava - sentivo de dar loro qualche forza era in me" (p. 121). Es la ocasión para despedir a los idiotas y saludar a los normales. Ciao!: "Tutti lo ripetavano: troppo c'era ancora di fare pei fanciulli normali!" (p. 125). Y es que el idiota, que no atiende, que no repite, que no puede, sólo es capaz de succionar y, "ya que nos referimos al lactante y al pecho, y como la lactancia es la succión, digamos que la pulsión oral es el hacerse chupar, es el vampiro" (El Seminario de Jacques Lacan. Libro 11. Los cuatro conceptos fundamentales del psicoanálisis. 1964. Texto establecido por Jacques-Alain Miller. Traducción de Juan Luis Delmont-Mauri y Julieta Sucre. Buenos Aires - Barcelona - México: Ediciones Paidós 2005, p. 203). Cfr. J. M. VÁzquez-Romero, "Dialécticas idiotas (entre salvajes y zombis)": Pensamiento, vol. 74 (2018), pp. 853-882.
} 
tete-que es como decir siempre y/o nunca, ya que el érase del entonces sería el érase una vez (un cuento, un mito, ¿el freudiano?: Die Triebenlehre ist sozusagen unsere Mythologie, "la doctrina de las pulsiones es, por así decirlo, nuestra mitología»), esto es, más que el escorzo del objeto perdido en aquel entonces, la mise en abyme que el corte mismo produce en un entonces y el entonces mismo que produce el corte ${ }^{3}$.

Sin embargo, la fijación del impulso, la fissità sopra un oggetto, la polarizzazione dell'attenzione, se representará y se organizará pedagógicamente no como una compulsión a la repetición, en la que el impulso se empeñara en trabarse en torno a un vacío de una ausencia, sino como una impulsión espontánea que se articularía armónicamente al topar en el ambiente con el objeto predestinado: "quelche cosa di organizzato in rapporto diretto alla sua organizzazione interiore [...] come l'insetto libero deve trovare nella forma e nella qualità dei fiori una corrispondenza diretta di forma e di sostanze" ${ }^{4}$. Interior y exterior conformarían un todo, una compleción y completitud entre tendencia y medio que redundaría en el desarrollo del individuo, de modo "[...] parangonabile a quello che rende il neonato capace di succhiare il latte alla mammella, la quale nella sua forma esterna e nella sostanza elaborata, corrisponde perfettamente ai bisogni di bambino" 5 .

En definitiva, la confianza en un método que pueda absorber metódicamente el plus de la repetición, plus, no olvidemos, generado por un minus.

\footnotetext{
3 “... le sein nourricier de la mère ne suffira jamais à satisfaire définitivament sa pulsión orale” (R. BERnET, Force - Pulsion - Désir. Une autre philosophie de la psychanalyse, Paris: Libraire Philosophique J. Vrin, p. 235. En nota se cita el siguiente pasaje de Le Séminaire, libre XI lacaniano (Paris: Seuil 1973): "Car si on distingue [...] le besoin de l'exigence pulsionelle - c'est justement parce qu'aucun objet d'aucun [...] besoin ne peut satisfaire la pulsión", p. 153).

4 Il Metodo, p. 63.

5 Il Metodo, p. 64. Correspondencia, compleción, totalidad de sujeto y objeto, o del órgano del sujeto y del órgano del objeto, tanto que pareciera no haber sitio ni para el entredós de la fisura y del contacto en tal acoplamiento, del dentro en el fuera y del fuera en el dentro: ¿interior, exterior? Pero ¿es posible olvidar el corte?: "¿de qué lado está el seno? ¿Del lado del que chupa, o del lado del que es chupado? Hay una ambigüedad, de la que la teoría analítica ha hablado a veces a propósito del seno y de la madre, subrayando, por supuesto, que no son lo mismo" (El Seminario de Jacques Lacan. Libro 10. La Angustia. 1962-1963. Texto establecido por Jacques-Alain Miller. Traducción de Enric Berenguer, corrección de Gabriela Ubaldini. Buenos Aires - Barcelona - México: Editorial Paidós 2010, p. 181). Si hubiera tal corrispondeza diretta, perfetta, ‘̨a qué la repetición, esa compulsión que circula a lo largo del perímetro de un vacío, de aquella teta del érase una vez? No obstante, “... the drive’s goal—to reach its object-is «false», it masks its «true» aim, which is to reproduce its own circular movement by way of repeatedly missing its object [recordemos, 44 veces]. If the fantasized unity with the object would have brought the full/impossible incestuous jouissance [succhiare la mammella], the drive's repeated missing of its object does not simply compel us to be satisfied with a lesser enjoyment, but generates a surplus-enjoyment of its own, the plus-de-jouir" (S. ŽIžEK, Sex and the Failed Absolute, London - New York: Bloomsbury Academic 2020, p. 294). Será el plus-de-jouir generado en la actividad el que se pretenderá habilitar educativamente, en términos no de mera utilidad (the drivés goal), sino de precisión, perfección (en la inhibición): la absorción pedagógica del plus (cfr. infra).
} 
La espontaneidad del desarrollo del niño estaría orientada a la consecución de los medios necesarios para su desarrollo, o la susodicha conjugación de tendencia y objeto, la cual presupondría la exteriorización o manifestación del impulso para cobrarse en el goce del objeto-pero exteriorización o manifestación ¿¿de qué?, ¿de quién?, pues no está en nada claro que, propiamente, hubiera ya algo o alguien ahí. ${ }^{6}$

Los objetos, las cosas, como la flor, como el seno, mejor, los senos, convocan el impulso, porque -a diferencia de la maestra que observa, ella también una centinela, una sentinella sempre all'erta, aunque pasiva y muda, pero ¿seguro que muda?nos dirigen su voz cautivadora:

La voce delle cose. - La maestra sorveglia, è vero; ma sono le cose di vario genere che «chiamano» i bambini di varie età. Veramente la lucentezza, i colori, la belleza delle cose gaie e adornate, sono altrettante «voci» che chiamano a sè l'attenzione del bambino e lo stimolano ad agire. Quegli oggeti hanno un'eloquenza che nessuna maestra potrebbe mai raggiungere: "prendimi», dicono; "conservami intatto, mettimi nel mio posto». ${ }^{7}$

Voz de la cosa, voces de las cosas, voces que no vocean, pero que parecen tener vocación de voz de la conciencia, que llaman, atrapando y articulando el impulso del niño, también dirigiéndolo y corrigiéndolo.

... tutto nell'ambiente è preparato in modo da rendere facile il controllo degli errori. Gli oggetti, dal mobilio, ai singoli materiali di sviluppo sono dei denunciatori, la cui voce ammonitrice non può sfuggire.

... Così che tutto l'ambiente è come un educatore severo, una sentinella sempre all'erta: e ciascun bambino ne sente gli ammonimenti como se fosse solo dinanzi a quell'inanimato maestro.

(Voces y miradas de ese inanimado maestro, pero centinela, vigía, que, en ese auditorio y escenario, nos instan y reconvienen. Una auditoría y supervisión educativa que convendría interiorizar, muy a la manera del juez interior e innato kantiano, cuya vigilancia tampoco si può sfuggire, ya que nos sigue como nuestra sombra, es folgt ihm wie sein Schatten, y cuya voz no se puede apagar, zu hören kann er doch nicht vermeiden. ${ }^{8}$ )

\footnotetext{
6 "L'identification de "Je suis le sein" (dans la célèbre note posthume [de Freud]) désigne l'inassignable rapport d'un "Je» qui n'est pas un «Je»" (P. Lacou-Labarthe et J.-L. Nancy, La panique politique suivi de Le peuple juif ne rêve pas, Christian Bourgois éditeur 2013, p. 36).

7 Il Metodo, p. 276; ańadido en la 3a ed.: 1926.

${ }^{8}$ Cfr. I. Kant, Tugendlehre $\$ 13$, «Von der Pflicht des Menschen gegen sich selbst, als den angeboren Richter über sich selbst».
} 


\section{$[\ldots]$}

E il fanciullo obbedisce a quell'oggetto che corrisponde in quel momento al suo più vivo bisogno di azione. Così in un campo, i petali di tutti i fiori chiamano altre vite coi loro profumi e coi colori, ma l'insetto sceglie il fiore che è fatto per lui. ${ }^{9}$

(Aqui, en este ambiente, la lección y reconvención, ammonimento, del objeto espabilan y fijan mi impulso en una corispondenza como la del insecto y la flor: polinización. $\left.{ }^{10}\right)$

Así, pues, voces que al unísono proscriben y prescriben el gozo, a cuyas advertencias no podemos sellar nuestros oídos-y, entretanto, la maestra, pasiva y muda, sorveglia, vigila y supervisa.

Stabilitosi il fenomeno della concentrazione sopra un lavoro, noi dobbiamo sorvegliarlo con scrupolosa esatezza, graduando gli esercizi secondo l'esperienza suggerisce. "Il nostro sforzo di maestre per stabilire la disciplina è di applicare rigorosamente il metodo».

Di qui già resulta la grande difficoltà di disciplinare veramente l'uomo. Non è con la parola, che si ottiene: nè l'uomo si disciplina «udendo un altro parlare»... ${ }^{11}$

\footnotetext{
${ }^{9}$ Il Método, pp. 323s.
}

${ }_{10}$ Pero la correspondencia nos podría llevar muy lejos, devenir orquídea de la avispa, avispa de la orquídea: "En la línea o el bloque de devenir que une la avispa y la orquídea se produce una común desterritorialización, de la avispa en tanto que deviene una pieza liberada del aparato de reproducción de la orquídea, pero también de la orquídea en tanto que deviene el objeto de un orgasmo de la avispa liberada a su vez de su propia reproducción" (G. Deleuze, F. Guattari, Mil mesetas. Capitalismo y esquizofrenia, traducción de José Vázquez Pérez con la colaboración de Umbelina Larraceleta, Valencia: Pre-Textos 2004, p. 293). ¿Y el niño y el material, o un juguete? Un caballito de madera: Warum war das nicht Lüge, wenn man dies / "Pferd» nannte? Weil man selbst ein wenig / als Pferd sich fühlte mähnig, sehnig, vierbeinig wurde - (um einmal ein Man zu werden?) Aber war man nicht ein wenig Holz zugleich um seinetwillen und wurde hart im Stillen und machte ein vermindertes Gesicht? - ¿Por qué no era mentira cuando a aquello / se le llamaba "caballo»? Porque uno mismo se sentía / un poco caballo, iba teniendo crines, tendones, / cuatro patas... (¿para convertirse alguna vez / en un hombre?) ¿Pero no se era / al mismo tiempo un poco de madera por amor a él / y se endurecía uno en secreto / y se ponía una cara reducida (de R. M. Rilke, Requiem auf den Tod eines Knaben, Réquiem a la muerte de un niño, trad. J. MunáRriz). Deveenir-caballo, devenir-hombre, devenir-madera...

${ }^{11}$ Il Metodo, p. 661. La maestra sorveglia, è vero. "Sorvegliàre», del fr. surveiller («sor», fr. sur, lat. supra, y "vergliare», "far la guardi», fr. veiller, lat. vigilare ("nam et grace quod dicitur episcopus, hoc latine superintentor interpretatur: qui superintendit, qui desuper videt”, Agustín, Enarrationes in Psalmos, 126, 3). La maestra vigila, como una psicóloga, porque, una vez que la relación educativa se establece entre nińo y material, queda en franquía, y espía y registra. De ahí la posibilidad de una bío-educación, analogía o especificación de la bío-política foucaultiana: si se puede hablar de una estatización de lo biológico cuando el poder estatal recae sobre el ser viviente (cfr., por ej., Il faut défendre la société. Cours au Collège de France, 1976, clase del 17 de marzo), podríamos referirnos a una pedagogización de lo biológico, cuando el poder educativo recae sobre el ser viviente (impulso, espontaneidad, motivación, desarrollo, crecimiento, formación, etc.). El viejo maestro deja caer el látigo y el libro de sus manos, los atributos de su poder y autoridad, y la joven maestra que lo releva se convierte en fata invisibile, que con su toque mágico todo lo anima, hasta mantener la llama del pebetero interior del niño: "ciascuno deve trovare la possibilità di «raccogliere» in se stesso e nell'attività calma e silenziosa che non ha uno scopo esteriore come fin ma ha lo scopo di mantenerse accesa quella fiamma interiore alla queale si riatacca la nostra vita" (Il Metodo, p. 662). 
("Altro parlare», "otro hablar», distinto de la lección, precepto, reconvención magisterial, un hablar alternativo que parece hablar para "altro udire», "otro oír, otro escuchar».

Y, no obstante, ¿no será ella, la maestra, quien preste, disimuladamente, su voz al objeto, por arte de ventriloquia, y asi separe su mandato de la enunciación para invisibilizar su mirada? ${ }^{\text {?2) }}$

El impulso se exterioriza, esforzándose por exponerse al objeto (fissità, polarizzazione) y responder a su llamada sobrecogedora; y, a la vez, las comandas de ese ambiente preparado se interiorizan sumisa y gozosamente, por parte del niño. Una circulación que parece querer refigurar la relación amboceptora de boca y pecho.

El objeto consigue que ese impulso circule en el circuito pedagógico, fijado, polarizado, imantado, pero se corre el riesgo de que el bólido pueda derrapar y salirse de la vía por exceso de velocidad. El método pedagógico ha de regular el exceso pulsional, que acecharía insidiosamente al proceso educativo, abocándolo a una actividad inútil y perjudicial,

... come p. es. tirare tutto l'incastro solido come un carrettino; o costruire casette con le spole di seta dei colori, o camminare sulle aste messe in fila, o mettere un telaio da allacciature intorno alla testa come una collana e via dicendo. Uso abusivo del materiale che rispondendo ad un disordine, o a bisogni diversi da quelli che il materiale può soddisfare, lo inutilizzano: e ne consegue dispersione di energia, chiasso: tutte azioni che allontanano il bambino dalla possibilità di concentrarsi, e quindi dal fine di migliorare e di sviluppare. È allora come se una emorragia fisica disperdesse quel liquido sanguigno che deve concentrarsi nel cuore per mantenere la salute e la vita. ${ }^{13}$

Desinhibición, dispersión, desviación y desbordamiento de la vida, potlach, emorragia, esplosione del movimiento del impulso, diríamos motu proprio que no parece

\footnotetext{
12 “... Lacan l'illustre d'une manière tout à fait singulière: «la voix dont un texte au moins de la Bible nous dit qu'elle se fit entendre au peuple parqué autor du Sinaï, non sans que cet artifice y suggère qu'en son énonciation elle lui renvoyait sa propre rumeur, les Tables de la Loi n’en restant pas moins nécessaires à connaître son énoncé». On aura compris que ce que vise ici Lacan est la voix qui, exemplifiant la disjonction du sujet de l'énoncé et du sujet de l'énonciation, revient de l'Autre sur le sujet - en l'ocurrence, le peuple rassemblé. C'est la logique de la voix comme objet $a$. [.../...] d'une part, l'objet a est bien ce qui fait en quelque sorte interface entre le symbolique et le réel (c'est sa fonction d'objet chosique de faire à la fois relation et disjonction entre le désir et la Chose), et si, d'autre part, le Surmoi est ainsi associé de manière essentielle à cet objet $a$, alors ce Surmoi doit être en quelque façon indexé sur la catégorie du réel. Et, à ce titre, il engage la jouissance. Plus précisément: il est impératif de la jouissance" (B. BAAS, "Ulysse en Baltique": Filozofski vestnik, volume XXXV, number 2, 2014, pp. 22s.).

13 Il Metodo, p. 440. Y entonces sí, entonces “... l’autorità della maestra soccorre la piccola anima pericolante, porgendole ora dolce, ora energico aiuto" (id.).
} 
tener término, que no se fija, que no se apacigua - que no se justifica, aunque su objeto sea didáctico, ya que su meta no lo es. Por ejemplo, la explosión de la escritura:

L'esplosione della scrittura.

Il bimbo mi guardò, sorrise, stette un momento come per esplodere in qualche atto di gioia, poi gridò: «scrivo! Io scrivo!» e chinato in terra scrisse sul pavimento mano, quindi, entusiasmato, scrisse ancora: camino, por tetto. Mentre scriveva continuava a gridare forte: «scrivo! so scrivere!» tanto che alle sue grida accorsero gli altri bambini e gli fecero circolo guardando stupiti. Due o tre mi dissero frementi: «il gesso; scrivo anch'io» e difatti si misero a scrivere varie parole: mamma, mano, gino, camino, ada.

Nessuno di loro aveva mai preso in mano un gesso o un qualsiasi istrumento per scrivere: era la prima volta ch'essi scrivevano...

Il bambino che scriveve per la prima volta una parola, era in preda a gran gioia; io lo paragonai subito alla gallina che ha fatto l'uovo. Infatti nessuno poteva ripararsi dalle chiassose manifestazioni del piccino; egli chiamava tutti a vedere, e se alcuno non si muoveva, lo pigliava pel vestito costringendolo a venire: era necesario che tutti andassero là, a mettersi intorno alla parola scritta per ammirare il prodigio, e per unire le loro esclamazioni di meraviglia, alle grida di gioia del fortunato autore. Pero lo più questa prima parola era scritta in terra: e allora il piccino si metteva in ginocchio, per essere più vicino all'opera sua, per contemplarla più inmediatamente.

Dopo la prima parola, il bambino continuava a scriver ovunque, per lo più sulla lavagna, con una specie di frenesia: io vidi i bambini agglomerarsi attorno alla lavagna per iscrivere e dietro ai piccini in piedi, formarsi un'altra fila di bambini montati sulle sedie, che scrivevano al disopra dei primi, e altrettanti al di dietro della lavagna: vidi altri fanciulli rimasti fuori, ricorrere a sgarbi, a dispetti, rovesciare le seggioline su cui erano in piedi i compagni, per trovare un poco di posto, e infine i soccombenti alla lotta chinarsi in terra e scrivere sul pavimento, o correere verso gli sportelli delle finestre e verso la porte, empiendole di scrittura.

[...]. In famiglia avveniva lo stesso; e alcune madri, per salvere il pavimento, e perfino il pane sulla cui crosta trovano parole scritte, dettero ai loro bambini della carta con un lapis. ${ }^{14}$

(Escribo, escribo como un poseso, como una máquina, aqui y allá, arriba y abajo, en la pizarra, pero también en puertas y ventana, suelos y techos, subiéndome a las sillas, metiéndome en broncas, grafiti hasta en la corteza del pan... itatuaré mi piel?)

Pero aquí, a diferencia del fatto fondamentale, la compulsión a la repetición -esa logorrea o graficorrea tan gozosa- resulta del todo inconveniente, por excesiva, y hasta agresiva, para el método educativo (pero ¿no resultaban excesivas aquellas 44

$\overline{14}$ Il Metodo, pp. 556-558. 
repeticiones?). La educadora reaccionará para reducir y adaptar el acontecimiento educativo, ese excedente excesivo de la misma repetición.

Se separa el mecanismo motor de la escritura, $i$ meccanismi motori, de la función cognitiva que supone, che rappresenti un lavoro vero e proprio dell'intelligenza ${ }^{15}$, con lo que se inactiva la atracción de la flor, del pecho, del objeto, para así poder perfeccionar, a su debido tiempo, el automatismo, sin todavía escribir, o, dicho de otro modo, para que sea sólo la mano la que escriba y la que goce escribiendo:

Bisogna andare indietro e cercare una mano [ed. 1950: indietro per trovare una mano] infantile ancora incoordinata «morbida nella funzione»: è la piccola mano cercante del bimbo piccolissimo di quattro anni che «tocca tutte le cose intorno» nella ricerca irresistibile e inconscia di [ed. 1950: nel tentativo irresistibile e inconscio di] stabilire le sue coordinazioni definitive. ${ }^{16}$

La función (escritura) provocaría por sí misma la disfunción o explosión de la repetición, que su objeto acabe por ser la repetición misma, dando lugar a esa exaltación y a ese frenesí que todo lo saca de quicio, lo pone fuera de sí (esa gran gioia o plus de gozo ${ }^{17}$. La intervención didáctica invierte el proceso al separar el impulso, disociándolo de su presunta finalidad, para negar su negatividad o esplosione $e^{18}$.

Se persigue contener la hemorragia del gozo por medio de una ascesis que pareciera perversa, por cuanto se sirve de esa misma negatividad que afloja y desliga el impulso del objeto o la causa del efecto: por un lado, scrivere, y por otro, scrittura. Esa separación permitiría mecanizar, maquinizar, el impulso sin extinguirlo y obtener una repetición que ya no será explosiva, sino implosiva.

La vera scritura è una prova, è lo sfogo di un impulso interno, è il compiacimento di esplicare un'attività superiore: non è un esercizio. E come pei mistici l'anima si perfeziona con la preghiera, cosi pei nostri piccoli, l'espressione più alta della civiltà umana, il lin-

${ }_{15}$ Cfr. id., p. 522. "Questa preparazione così remota e indirette è una preparazione della mano a scrivere, non è una preparazione della scrittura: e la due preparazione non si devono confondere tra loro" (p. 526, n. 251, sg. ed. 1950).

16 Id., p. 524 , n. 251.

17 “... la pulsion excessive ou pulsion de répétition est le vrai visage de cette énergie pulsionnelle neutre, «indifférente» et «versatile», dont, selon Le moi et le ça se nourrisent et les pulsions de vie et les pulsions de mort. [...]. C'est seulement dans la com-pulsion de répétition que la pulsion se montre dans sa verité toute nue" (R. BERNET, Force - Pulsion - Désire, cit., pp. 267 s.).

${ }_{18}$ El porqué del efecto subversivo de la pulsión no consistiría sólo en que su objeto acabe siendo la satisfacción misma, sino en su intrínseca negatividad o estado de carestía, desconcierto del impulso mismo. “... that satisfaction becomes object (start to function as the object of the drive) [la esplosione della scrittura] only because it give body to this negativity [el vacío o hiato que separa la pulsión de cualquier objeto, y hasta de sí misma...], and not simply as satisfaction for the sake of satisfaction [.../ The drive does not want (us) to enjoy. The superego wants (us) to enjoy [...] actively blocking access to negativity that drives it" (A. Zupančič, What is Sex, Cambridge, Mass. - London: The MIT Press 2017, p. 104). 
guaggio grafico, si acquista e si eleva con esercizi sempre uguali in atti, che non sono la scrittura. ${ }^{19}$

Una compleja operación en la que los medios (pregare, scrivere) subliman (elevazione, innalzamento; cfr. infra) el fin (contemplatio, scrittura), medios sin los cuales el fin no alcanzaría la expresión più alta. Se trata de conseguir que el ejercicio reglado, más que ordenarse a la acción y a la producción, se haga forma de vida, "... allontanando - de tal modo- dall'illusione che il successo raggiunto basti..." ${ }^{20}$ : prova e sfogo de la interioridad. Una forma de vida, forma de ser, con los principios de su regla:

Essi divengono virtuosi perchè esercitano la pazienza -ripetendo gli esercizi-, la mansuetudine cedendo al comando, al desiderio degli altri -, la bontà godendo del bene altrui, non sentendo invidia nè emulazione; fanno il bene nel gaudio, nella pace; e sono eminentemente, meravigliosamente laborios $i^{21}$.

Y más que disciplinar el gozo, se conseguiría gozar de la disciplina-bloqueando así, presuntamente, en virtud de su propia impulsividad o energía, la negatividad del impulso (pulsión, Trieb).

Avere imparato pel bambino è un punto di partenza; quando ha imparato, allora comincia a godere della ripetizione dell'esercizio e ripete un numero indefinito di volte ciò che ha imparato, con evidente soddisfazione: egli gioisce di esercitarsi, perchè con ciò sviluppa le sue attività psichiche.

$[\ldots]$

[...] nella ripetizione, non nell'apprendimento, consiste l'esercizio che sviluppa la vita. ${ }^{22}$

(La conversión milagrosa del impulso: “... una specie di miracolo avvenute nella vita interiore di ciascun bambino.[/] Si pensiamo agli adulti, ci viene in menti il fenómeno delle conversioni..." 23 .Por qué se goza (de) la repetición? ¿Se logra en ella una continuidad que soporta la identidad? Si asi fuera, esa repetición habría de estar siempre fijada, apaciguada ya como respuesta, en vez de explotar, despilfarrándose, sublevándose.)

19 Il Metodo, pp. 561 s.; si bien en la ed. de 1950 eliminó «che non sono la scrittura».

${ }^{20}$ Id., p. 562.

${ }^{21}$ Id., p. 672. Una regla religiosa, por lo menos más religiosa que escolar; más interior que exterior: “... una forma di disciplina indiretta [...]. Essa sottintende un concetto della vita generalmente noto più nei campi delle religioni che in quelli della pedagogia scolastica - perchè si rivolge alle energie interiori dell'uomo..." (Il Metodo, p. 673; doy la lectura de la segunda ed.).

22 Id., p. 666. "La disciplina dunque non è un fatto, ma una via, sulla quale il bambino conquista, con precisione che potrebbe dirsi scientifica, il concetto della bontà" (id., p. 663).

23 Id., p. 661. 
El ejercicio tramita la repetición como fin en sí, vita, que no por su utilidad, non nell'apprendimento, suturando la herida de la pulsión y remitiéndola a una necesidad que se presume biológica, pero se estipula axiológica por la intervención educativa (deve rispondere), pues no tarda en comparecer la educadora, como operadora del superyó o unidad sintética, para cubrir el vacío del impulso y adjudicarle una necesidad indeterminada y gozosa, o, dicho de otro modo, prescribiendo la repetición como retorno a la necesidad y de la necesidad-pero ya no en corispondenza, co-rispondenza, sino en rispondenza.

Como consecuencia, "...quando il fanciullo è riuscito a raggiungere questo stato, di ripetere un esercizio, egli è nella via di sviluppo della sua vita, e si manifesta esternamente come disciplinato", pero -como se nos advierte a continuación- subsiste el riesgo de que esa energía se derrame y desparrame, y por ello "... la ripetizione deve rispondere a un bisogno..." 24 .

Por lo demás, tratándose de un fanciullo bisognoso, menesteroso, falto, ¿cómo no va a responder?

Una economía exenta de la limitación de lo útil, pero una economía todavía más restringida, que ajusta la repetición que ajusta el impulso a una supuesta necesidad basal, biológica, que estipula el supuesto saber de la pedagoga y que no cede a la compulsión impropia, sino que se acompasaría al ritmo de la vida, de l'evoluzione naturale ${ }^{25}$. "Infine, c'è il periodo di sviluppo compiuto, consistente in ciò: che quando si sa fare una cosa, resta permanente la capacità a riprodurla" ${ }^{26}$. Pero si se trata de capacità (dynamis) de repetir, subsistirá e insistirá, también, la posibilidad de aquella eventualidad de la repetición excesiva del objeto (lúdica, frenética, maníaca...), que acaba siendo equivalente a la potencia de la sublevación. Como reacción "qui sta il metodo sperimentale dell'educazione: occorre offrire gli esercizi rispondenti alle necessità di sviluppo dell'organismo..." ${ }^{27}$.

Frente al exhibicionismo de la repetición diseminadora, la capacidad, la capacità, puede y es capaz de retenerse, restar en sí y restarse a sí como inactividad, inhibición, capacidad-de-no, potencia-de-no (por tanto, no su reducción a cero).

¿Estará ahí la clave de la disciplina interior, indiretta?

Pues -sigue advirtiéndonos- no se ha de confundir la disciplina externa con la disciplina interior ${ }^{28}$. La auténtica disciplina demanda su ejercitación para la elevación, y de ese modo gozarse en la repetición, o saber obedecer: “... perfezionan-

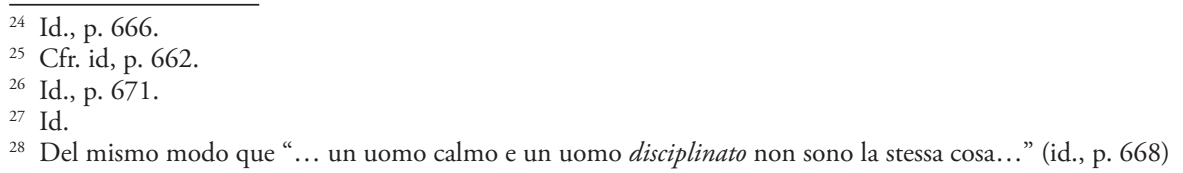


dosi negli esercizi, nasce nel bambino la gioia di sapere obbedire" ${ }^{29}$, una alegría espontánea, como lo manifestaría “... l'espansione con cui abracciono le ginocchia della maestra, o con cui attirano in basso le sue spalle e la sua testa per baciarla in viso..." 30 "Para besarla en el rostro». ¿Cuántas veces?

Contención y elevación y hasta sublimación del impulso, hasta “... trovarsi in un mondo superiore" ${ }^{31}$, por medio de la conversión que implosiona la explosión (polución espiritual que se comparaba, recordemos, a una emorragia fisica), invirtiéndola y revertiéndola en valor interior o, mejor, plusvalía íntima.

Tal habilitación explicaría la minuciosidad metódica, que rebasa la funcionalidad y se obsesiona, casi perversamente, decíamos, por la precisione, la esatteza.

... sotto l'attività che li [i bambini] dirige a raggiungere vari scopi pratici, esiste uno speciale segreto di successo: è la precisione, l'esattezza con cui gli atti si devono compiere $[\ldots]$.

Il movimiento generico è una funzione rozza, ma se in esso si innesta un motivo di perfezionamento, esso cresce di valore: le mani allora non si lavano più solo per averle pulite, ma sopratutto per acquistare l'abilità di lavarle a perfezione. [...] l'imparare cose pratiche è solo un richiamo esterno, è il motivo apparente che stimola un bisogno profundo di organizzazione. ${ }^{32}$

(El interés y la utilidad, scopi pratici, cose pratiche, los motivos, funciones que regulan y restringen económicamente la actividad educativa, son aparentes, epifenómenos bajo los cuales calienta esa profunda necesidad de organización, ese bisogno profondo: precisione, esattezza.)

Una didáctica rayana en lo obsesivo y que persigue poner al margen lo que se pasa de la raya, en dique seco todo lo que se derrama y en silencio cualquier tilín. Es como si aquella voz de las cosas se hubiera convertido en voz en off o como si se oyera sin que sonara siquiera, para no distraer la atención del niño, y sólo se oyera ya dentro como un ordenamiento interior de la voluntad, que se goza masoquistamente al negarse (intatto, nel mio posto):

Il filo. - [...] un mezzo che potesse aiutare i piccoli bambini a render sicuro l'equilibrio della persona, e insieme a perfezionare il movimiento che è fra tutti fondamentale: cioè il camminare.

\footnotetext{
29 Id., p. 672.

${ }^{30}$ Id., p. 660.

31 Id., p. 664.

32 Id., p. 277; añadido en la $3^{\text {a }}$ ed.
} 
Disegnata una línea a forma di lunga elisse [sic.] sul pavimento [...] vi si cammina mettendo il piede intieramentee [sic.] sulla linea, cioè in modo che questa sia lungo l'asse della pianta del piede. La collocazione esatta del piede [...]: la punta ed il tallone sono entrambi sulla linea. [...] Quando il bambino comincia ad assicurare la sua deambulazione, gli si insegna a superare una ulteriore difficoltà: i piedi devono avanzare in modo che l'anteriore posi col tallone a contatto della punta dell'altro piede. L'esercizio comporta non soltanto lo sforzo di mantenere l'equilibrio, ma esige da parte del bambino una intensa attenzione per dirigire i piedi nella posizione dovuta. ${ }^{33}$

Esercizi concomitanti. - [...]. I camminatori sul filo, dunque appena hanno superato le prime difficoltà, e raggiunto l'equilibrio, possono prendere una di quelle bandierette [attraenti coi loro vivaci colori], purchè sappiano tenerla alta. Se con grande attenzione non controllano il braccio, la bandierina si abbasserà a poco a poco. [...].

Le difficoltà successive sono esercizi sempre più minuziosi di controllo dei movimenti; ecco una serie di bicchieri, che contengono liquidi colorati: il liquido è alto quasi all'orlo, e bisogna camminare tenendo ben diritto il bicchieri, affinchè il liquido non cada; tutta la mano dunque deve essere dominata dalla stessa volontà che dirige contemporaneamente i piedi[...].

Altri oggetti sono campane che bisogna prendere passando, e tenere immobilmente diritte, cioè perpendicolari: camminando tutto intorno al filo, non un suono si deve udire...

A questo punto c'è un interesse, che è nato, a superare sempre maggiori difficoltà: il fanciullino è lanciato in una ginnastica gioiosa $[\ldots] .{ }^{34}$

(Ejercicios para el niño montessoriano, camminatore sul filo, punta-tacón del funambulista que tensa sus brazos que sostienen banderolas, vasos y campanillas; ni una onda, ni una gota, ni un sonido. Blade runner. "La mano debe ser dominada por la misma voluntad que, a la vez, dirige los pies».)

Ocurre que el goce de tales actividades, ginnastica gioiosa, se retiene y contiene cada vez más, hasta que la actividad acaba negándose en la in-actividad, una didáctica rayana en lo histérico ${ }^{35}$ — como comprobaremos, "non si fa». ${ }^{36}$

\footnotetext{
33 Id., pp. 280s., ańadido en la $3^{\mathrm{a}} \mathrm{ed}$.

34 Id., p. 289, $3^{\mathrm{a}}$ ed.

35 “... somewhat ironically, psychoanalysis was born of imposible power, notably, the requirement to use no power. This came at the request of Frau Emmy von N.: «Don't move, say nothing, don't touch me», she told Freud at the beginning of treatment. Freud abandoned hipnosis and inverted the injuction to establish the setting for the talking cure with its famous rule, the prerequisite of this own (im)possibility" (D. Hece, "The Impossible Power of Psychoanalysis", en J. Clemens and R. Grigg, editors, Jacques Lacan and the Other Side of Psychoanalysis, cit., p. 217).

36 "Authority, which, once again, is not the same as power or force, prepares the act, if it is an act, of extreme renunciation" (A. Ronell, Loser Sons. Politics and Authority, Urbana, Chicago and Springfield: University of Illinois Press 2012, p. 29).
} 
La ejecución correcta de la actividad sería sólo el accidente de la substancia, la apariencia de la esencia educativa, que es la ordenación interior del impulso: “... l'imparare cose pratiche è solo un richiamo esterno, è il motivo apparente che stimola un bisogno profondo di organizzazione" ${ }^{37}$. El objeto quiere sitiar y situar mi impulso, pero el meollo no es la ob-jeción del objeto (problema), sino la sub-jeción del objeto (emblema), su interiorización, por lo que la objeción del objeto habrá de ser sujetada en el sujeto- pero eso si es que tiene sitio donde colocarse o sujetarse (mise en abyme?). ${ }^{38}$

El objeto cae en el interior después de tanta repetición, pero su voz manda dentro: zitti e fermi! Callado y quieto el impulso que era el resorte, el cual, aunque convocado como "... [il] misterioso impulso ad agire, che è tutto il fondamento del progresso [del bambino]" ${ }^{39}$, tendrá, pues, que ser silenciado y refrenado. Pero de modo que se inhiba a sí mismo, teniendo, para ello, que diferenciarse en sí mismo y reconocerse como otro, que, ¿̇finalmente?, habré de hacer mío. Sin duda, no haciéndome suyo, sino empoderándome de él, lo que exigirá mi dividuación, condición de que sea dueño de mí mismo, padrone dei propri atti ${ }^{40}$.

De ahí la deriva, cada vez más implacablemente quietista de los ejercicios que pretenden esercitare, formare la propia volontà, invirtiendo así aquella ascensión al mundo superior en descenso que vacía el tiempo:

Immobilità e silenzio. - Un tutt'altro genere di esercizi di controllo sui movimenti, è quello che conduce a render possibile il silenzio assoluto. Non è un approssimativo stare zitti e fermi; ma è una perfezione raggiunta gradualmente, nel non emettere alcun suono, nel non produrre il più piccolo rumore, che può farsi muovendo un piede, o strisciando una mano, o respirando sensibilmente. Il silenzo assoluto equivale ad assoluta immobilità. ${ }^{41}$

(Callados y quietos, zitti e fermi. Pero esa inmovilidad absoluta, del pie, de la mano, del aliento, que equivale a ese silencio absoluto, ¿apaga o apacigua las sistole y diástole del piccolo corazón?)

\footnotetext{
37 Id., p. 277. “... il bambino ha in prevalenza lo scopo inconscio di sviluppare se stesso. Perciò disprezza tutto che è raggiunto e ama ciòche è da raggiungere” (id., p. 666).

38 "L'intime est toujours plus au fond que le fond le plus profond (c'est aussi pourquoi il touche à l'extime, ce terme forgé par Lacan—ou bien je dirais qu'il s'extime...). Mais aussi, l'intime est toujours le lien d'un partage - de soi et de l'autre" (J.-L. NANCY, L'«ily a» du rapport sexuel, Paris: Éditions Galilée 2001, p. 45). Y más que fundar al sujeto, lo desfonda, para exponerlo, expósito, a la convocatoria del otro, aquella ventrílocua de la cosa. “... il faut dire qu'il n’y a pas, à parler proprement, d’object du désir. Ce que le désir désire ne lui est pas ob-jeté, n'est pas placé devant lui comme en face de lui, mais fait partie de son mouvement désirant”, a lo que hay que añadir que "la chose du désir n'est pas plus ob-jectivable que sub-jectivable. Elle n'est pas «-jetable » du tout : ni objet perdu ni sujet d'une quête, mais le jet lui même ou la jetée, l'envoi, l'adresse, l'inter-jection" (id., pp. 34s.).

39 Il Metodo, p. 223, n. 22; ed. 1926.

${ }^{40}$ Cfr. id., p. 277.

${ }^{41}$ Id., p. 289, n. 48; ed. 1926.
} 
Ese reposo que no es inerte, sino resistente, y ese silencio que no es tácito, sino transitivo, comprometen al impulso y resultan en un equilibrio extático, no entre fuerzas con sentidos opuestos (activo versus pasivo), sino por el repliegue de la misma fuerza al convertirse en obstáculo para sí (inhibición), que, lejos de anularse en ese conflicto consigo en una oposición real por la que se redujera a cero, se superaría en una oposición dialéctica (disciplina). ${ }^{42}$

El silencio, activo como es, algo calla (un segreto?), pero, entretanto, deja oír, o consuena con altro parlare que disciplina con la disciplina attiva propia de aquel que es padrone di se stess ${ }^{43}$. Silencio absoluto de quien se silencia y se hace silencio y, a la chita callando, se dice no (nulla, niente) a sí mismo, se stesso, y, merced a ello, se eleva più alto hasta un estado superior, con "l'orgoglio interno di aver superato se stesso" ${ }^{44}$.

Superación de quien se releva y eleva, por sí mismo, a sí mismo, inhibiéndose ${ }^{45}$ :

42 "Comme la dunamis dans la Physique aristotélicienne, la pulsión freudienne présse encore quand elle fait semblant de se reposer" (R. Bernet, Force - Pulsion - Désir, cit., p. 231). Por tanto, la inhibición no indicaría en absoluto una supresión del impulso, todo lo contrario: una hipertensión que dirige su curso hacia el interior. "Il [Freud] mentionne aussi la possibilité de pulsions «inhibées quant au but», c'est-à-dire de pulsions dont le cheminement est, en cours de route, dévié sur une autre «voie» et vers un autre but” (id., p. 233).

43 Cfr. Il Metodo, p. 191.

44 Id., p. 664.

45 “... il silenzo può intendersi in modo positivo - come uno stato «superiore» al normale ordine delle cose. Come una inibizione instantanea che costa uno sforzo - une tensione della volontà...” (p. 420, n. 248, ed. 1926). Inibizione: "inhibitio, -onis [inhibeo], f., acción de remar hacia atrás...”; “inhibeo [...] [in, habeo], tr. Mantener en, retener, sostener, detener, contener, reprimir: equos i., frenar los caballos; aliquem i., detener a uno; impetum i., detener un impulso; facinus i., impedir una mala acción; verecundia inhibemur... credere, el pudor nos impidió creer || [término náutico] remar hacia atrás; remos i., remar hacia atrás || aplicar, emplear, usar: imperium i., ejercer su autoridad, su mando; suplicium alicui i., infligir a uno un suplicio; eadem suplicia i., infligir los mismos suplicios" (S. SEgura, Diccionario etimológico latino-español, Madrid: Ediciones Generales Anaya 1985). "In", ¿dónde y adónde el haber o los haberes de ese patrimonio y potestad del padrone? Si lo es di se, di se stesso - por tanto, de su impulso- de seguro que lo es privativamente, en lo privado, en privanza; en casi todas, o en todas sus acepciones, como iremos comprobando. "privativo, -a. 1 Propio y exclusivo de la persona que se expresa [...]. 2 Se aplica a las partículas y prefijos que expresan carencia; como "a-»." "privado, -a. 1 Participio adjetivo; falto de cierta cosa: 'Privado de inteligencia'. 2 Realizado o celebrado en la vivienda de alguien, en el seno de la familia o con asistencia limitada de unas pocas personas, y no en sitio público o para todas las personas de una colectividad amplia [...] (V. «ÍNTIMO, RESERVADO»). $\mathbf{3}$ "Particular». Se aplica a lo que se refiere a una persona como tal persona o como miembro de una familia y no como ciudadano o por su profesión: 'Vida privada, asuntos privados'. (V. «de incógnito, oficioso»). 4 (n. en masc.). Hombre que goza de la confianza de un soberano e influye en sus determinaciones. [...]. ${ }_{5}$ (n., en fem.) Retrete. 6 (id.). Excrementos depositados en sitio visible". "privanza. 1 Cualidad de lo que pertenece a la vida privada de alguien • "Intimidad". Vida que se desarrolla exclusivamente entre la familia. 2 Situación de privado o favorito” (M. Moliner, Diccionario de uso del español, Madrid: Gredos 1989). La privación de inteligencia nos remite al reverso obscuro del método, dise-

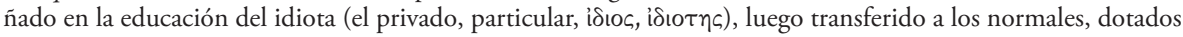
de impulso, de fuerza, de actividad (cfr. nuestra nota 2), pero quienes habrán de in-activarse (in-hibirse) para que, en esa torna que confina en la particularidad y privacidad de su retrete, hallar, en la excrecencia del vacío íntimo, el privilegio que me distingue ante los demás, o el haber en el deber ("PRIVAR, del lat. PRĪVARE 'apartar (de algo)', 'privar, despojar'. [...]: «cassare es vaziar y privar y castrar» [...]. Así en el sentido de 'apartado, particular', como en el de 'favorito, confidente' privado es ya frecuente desde Berceo...”, J. Corominas, A. Pascual, Diccionario crítico etimológico castellano e hispánico. Volumen IV, Madrid: Gredos 1989). 
... far loro sentire in perfetto silenzio, il tic-tac dell'orologio e tutti i piccoli rumori che sfuggono comunemente all'orecchio; e nel chiamare a uno a uno i piccini da una stanza vicina, pronunciando il nome di ciascuno a voce afona.

Per preparere tali esercizi, è necessario [hasta aquí sigo la lectura de las eds. de 1909 y 1913] insegnare ai bambini il silenzo: per questo faccio eseguire vari giuochi del silenzio [en ediciones de 1926 y 1950: vari esercizî di silenzio], che contribuiscono in modo notevole alla sorprendente capacità di disciplina dei nostri bambini.

Richiamo l'attenzione dei piccina sopra di me - che faccio silenzio.

Mi metto in varie pose: in piedi, seduta - immobile, silenziosa. Un dito che si muova, potrebbe produrre un rumore, sia pure impercettibile; potrei respirare in modo che si sentisse: ma no, tutto è assoluto silenzio. Non è cosa facile. Chiamo un bambino e lo invito a far come me: egli aggiusta in miglior posa un piede, ecco un rumore! [...].

[...] Essi [i fanciulli] mi guardano stupiti quando io mi metto in mezzo alla sala diritta, ed è veramente come se "non ci fossi». [...]. L'attenzione dei fanciulli è richiamata su ogni parte del corpo, in un'ansiosa volontà di raggiungere l'immobilità. [...]. I fanciulli restano affascinati da quel silenzo come da una loro reale conquista. "Ecco» - dice la direttrice "ora non c'è più nessuno - i bambini sono andati via» [ed. 1926: ora è tutto quieto come se non ci fosse più nessuno].

Raggiunto questo grado, si chiudono al buio le finestre - e si dice ai bambini: chiudete gli occhi - appoggiate lentamente la testa sulle mani - e mettete le mani spiegate a chiudere gli occhi. Essi si pongono così e torna nel buio l'assoluto silenzio. "Adesso ascoltate una voce leggera che vi chiama per nome». ${ }^{46}$

(Una reale conquista, conquista di se, di se stesso... pero jsi parece que no hay nadie!, ora non c'è più nessuno! No es extraño: tanta inmovilidad, tanto silencio. Habrá, pues, que llamar a los niños - ¿con un altro parlare? - por y en su nombre propio.)

Poco a poco la maestra, desde la sala vecina, sin asomarse, con la puerta apenas entreabierta, llama a cada uno por su nombre: “... chiamo a voce afona, strisciando le sillabe lungamente, como si chiamerebbe con chiaro grido a traverso le montagne..." 47 .

La voz de ella me llama por mi nombre, interpelándome por mi propio nombre propio, una voz que parece “... che giunga al cuore e chiami la loro anima” ${ }^{48}$, dando nombre propio, en propio, al impulso inmóvil, inhibido de su exhibición, casi inexistente, in-ek-sistente (ora non c’è più nessuno)__también in-ek-sistente?

Entonces me quieren como soy, es decir, como me llaman, como me nombran. ${ }^{49}$

\footnotetext{
46 Il Metodo, pp. 419-422.

47 Id., p. 422.

48 Id.

49 "In fact «called" is your most proper name, prior to any nomination, any baptism. This is why the call concerns you. Your being is being-called” (A. RonelL, Loser Sons, cit., p. 172).
} 
Ella me silencia, come se non ci fosse... più nessuno, niente, nulla, para entonces, adesso, darme ese nombre, con el que me reintegra como propio, y todo ese reconocimiento irá bien si, a la vez, silencio mi impulso, pero sigo esa llamada—si yendo a la zaga de esa llamada, silencio mi impulso para no provocarla; si silenciando mi impulso, oigo esa llamada con la emoción enarcada en la boca. Y, entonces, sí es mío mi impulso, cuando yo soy de la llamada: el envío de mí mismo, o la emoción de la pasión. Una vez que sigo emocionado y obediente a esa vocal que me llama por mi nombre, y hasta ella llego, reclinaré mi cabeza sobre su hombro, o su pecho, contenida la emoción.

E giunge alla porta con volto gioioso, fa qualche salto nella stanza vicina, soffoca piccoli scopî di risa; ovvero si attacca alle mie vesti appoggiando il volto al mio corpo [eds. 1926, 1950: si attaccava alle mie vesti appoggiandomisi addosso; o si poneva a]; o si pone a guardare i compagni che giacciono ancora nell'aspettativa silenziosa. Il chiamato sente quasi un privilegio, un dono, un premio. ${ }^{50}$

El descanso de la privanza en ese contacto íntimo y gozoso entre el deseo del nombre y el deseo de aquélla que nombra:

Allora mi accorsi che l'anima del fanciullo ha pur essa i suoi premî e i suoi godimenti spirituali. Dopo tali esercizî sembrava ch'essi mi amassero di più: certo erano divenuti più ubbidienti, più dolcemente miti. Infatti ci eravamo isolati dal mondo e avevamo passato qualche minuto insieme uniti tra noi; io a desiderli e a chiamarli - ed essi a ricevere, nel silenzo più profondo, la voce che si rivolgeva personalmente a ciascuno di loro, giudicandolo in quel momento il migliore di tutti. ${ }^{51}$

(Il migliore di tutti, el mejor, deseado y llamado, Yo, yo mismo, reconocido... y por mi nombre propio... ¿propio?)

Esa interpelación que me ha hecho temblar y arder, en mi privanza, por el deseo de obedecer, es la que enciende el espíritu, “... quella fiamma interine alla quale si riattaca la nostra vita". ${ }^{52}$

Pero en el trámite de esa interpelación acabaré perdiendo el nombre y el propio, mi representación, quedándome sin o con nada. ¡Acabaré oyendo el vacío en mi deseo, de mi deseo!

\footnotetext{
${ }^{50}$ Id., p. 423. "“Je suis une émotion»: le français oblige à cette amphibologie. Je vais à la suite d'une émotion, je la poursuis, je marche à sa traîne, dans son sillage - ou bien: je («moi»?) est une émotion, n’est que cela, ipse est le point incandescent, évanouissant, d'un trouble instantané" (J.-L. NANCY, La Naissance des seins, Paris: Éditions Galilée 2006, p. 13)

${ }^{51}$ Il Metodo, pp. 424s.

52 Id., p. 662.
} 
Deseo, mas, entonces, ni subjetivo, porque, bajo él, nada hay; ni objetivo, porque, frente a él, nada hay, ni nadie... ¿ ¿ni nadie? Eso pareciera, pues, ¿acaso se comparte el vacío?, ¿̇e comparte la nada?, ¿se comparte el zero? Porque, ¿qué me piden?, ¿qué me quieren?, che vuoi?:

- Tu caro, con le tue ditine mandami zero baci, «Tú, cariño, mándame con tus deditos cero besos» ${ }^{53}$.

Mandami zero baci: ¿se trata aquí de inhibir? Aun tratándose de ello (inhibire, in + habere, inhibire plus habere, en el interior, más bien hacia el interior, y tratándose de ello, hacia lo más interior, la intimidad), tal instrucción nos haría sentir la inhibición en su límite y al límite: cuando tengo, en privado, en privanza, ese cero, esa nada, ese nadie, que se me solicita (entonces, in - habere, in minus habere) ${ }^{54}$. ¿Cómo entender el mandar zero baci? ¿Es eso besar, besarse, baciare, baciarsi?

La sensación del límite en el que el deseo de darte, de darse, toca su límite y se toca en su límite, mucho más que si te diera, a ti, que me los pides, uno, o dos, o tres, o tantos como deseara $y / o$ desearas. La sensación del límite del deseo, cero,

53 ¿Qué son esos zero baci?, ¿qué es zero? Algo, ¿algo?, a la manera de la vuelta de tuerca lacananiana al dén, no "peut-être rien", sino "pas rien»: "Lacan takes up the coinage of a new word by Democritus, $\delta \dot{\varepsilon} v$, a strange entity which escapes the alternative between being and nothing: it is not a being but neither is a nothing, it gives existence to negativity [la negatividad aquélla de la pulsión], yet not by being something that one could identify or lay one's hands on or count as one $[\ldots / \ldots] \delta$ 'sv is also what is at stake in repetition, an entity which is being repeated without having a proper consistency or identity, a cause of the derailment of causality, but which cannot be give a separate ontological stature, an entity inhabiting a crack, not a positive being but not a nothing" (M. Dolar, "One Splits into Two": Das Magazin des Instituts für Theorie, 31, No 14/15, pp. 90 s.). Algo, ¿algo?, a la manera del asombro contrafáctico que el lema lacaniano Il n'y a pas de rapport sexuel nos provoca (“... moyennant notre sidération, que ce qu'il n’y a pas est autre chose que ce que nous savons bien avoir lieu”, J.-L. NANCY, L’ «il y a» du rapport sexuel, cit., p. 14). Algo, ¿algo?, algo así causa también la orden de mandare zero baci. Tal vez, más que por anti-performativa, porque en ella, como en el lema lacaniano, resuena la prohibición, la interdicción: "quelque chose de l'interdiction résonne ici [en el Il n’y a pas de rapport...], comme venant interrompre le couple qui baise (car nous avons aussi, de ce verbe, cet usage que je dirais réciproqual). Il se dessine une pragmatique performative de coitus interruptus. À ce compte, ce qu'il y a à comprendre ne serait que l'interdit. Et nous savons bien que c'est l'enjeu, en fin de compte (si l'on tient le rapport sexuel sur l'horizon de l'interdit de l'inceste)" (id., pp. 14 s.). Puede que esta última alusión nos despierte el recuerdo de aquella fusión de la boca del lactante con su, la mamella, metáfora fundacional del método. ¿Se trata, pues, de que no me den o me quiten, poco importa, de las manos, de la boca..., el uno, el dos, el tres..., tal como correspondería a un método educativo castrante, de que me conviertan en un cero a la izquierda? Pero, si "le mot «rapport» fait entendre une action, non une substance” (p. 16), ¿qué decir de con le tue ditine mandami zero baci?

54 "There is a well-known joke about Jews confessing their failures at a synagogue reunion. First, a mighty rabbi says: «Forgive me, god, I am nothing, not worthy of your attention!» After him, a rich merchant says: «Forgive me, god, I am a worthless nothing!» Then a poor ordinary Jew steps forward and says: «Forgive me, god, I am also nothing..." The rich merchant whispers to the rabbi: "Who does think he is, this miserable guy, that he can also say he is nothing?» There is a deep insight in this joke: to "become nothing» requires the supreme effort of negativity, of tearing oneself off from the immersion into a cobweb of particular determinations" (S. ŽIžEK, Sex and the Failed Absolute, cit., pp. 354 s.). 
nada, cero y nada, cero o nada, que se comparten, porque cero besos se piden, pero... ¿se dan?... Al menos se sienten:

Le lezione sullo zero. - Attendiamo che il bambino ci domandi segnando la casella dello zero: «e qui cosa bisogna metterci»? per risponderi: «nulla; zero è nulla».

Ma ciò non basta - occorre far sentire che cosa è il nulla. - Per questo usiamo degli esercizî che divertono immensamente i bambini. ${ }^{55}$

Se divierten tanto con esos ejercicios, immensamente, ¡tanto!, tanto que aquí el esercizio se convierte en giuoco, y, además, un giuoco della volontà, antes que un esercizio di numerazione ${ }^{56}$. Esa última, la numerazione, sería el motivo aparente, porque el objeto, el número, no es sino la ocasión de la meta: la volontá, la ordenación del impulso, de la pulsión (Trieb), o, mejor: mettere in attività i potere inhibitori. ${ }^{57}$

55 Il Metodo, p. 623. Nulla, nada, rien, “... que «rien» n'est autre chose que la chose même, res, qui a donné ce mot français qui désigne quelque chose, une chose quelconque, insignifiante, un détail, une vétille et qui ne devient «nulle chose» que s’il est lui-même nié («il n'y a rien») (J.-L. Nancy, Sexistence. Accompagné d'un frontispice de Miquel Barceló, Paris: Éditions Galilée 2017, pp. 60 s.). En italiano lo sobreentendido sería «la cosa», res, res nulla, y en español también, pero en este caso del lat. «res nata», de «nasci», nacer: “... cosa nacida, construido con oraciones negativas equivalía a "cosa alguna»: 'res nata non vidi', 'no vi cosa alguna'; convertido el adjetivo en pronombre por sobreentendimiento del nombre, daría en castellano «nada no vi»; y la atribución del mismo valor negativo a ambas palabras invitaría a evitar la repetición suprimiendo «no» cuando ambas estaban juntas: en vez de "nada no vi», "nada vi», con lo cual el pronombre "nada" pasó a tener constructivamente valor negativo por sí mismo, como el que tienen "cero" o «nulo"; ahora bien, en los casos en que "no" precede al verbo y «nada» le sigue, no se realiza la simplificación y subsiste, con aparente falta de lógica si se desconoce el significado etimológico de "nada», la duplicidad de negación. La consideración anterior es aplicable exactamente en la misma forma a "nadie, ninguno" y "jamás»" (Ma Moliner, Diccionario de uso del español, cit., art. nada; no obstante, cfr. J. Corominas y J. A. PAScual, Diccionario crítico etimológico castellano e hispánico, cit., art. nacer, donde se advierte de que el uso en frases negativas de RES NATA no es lo habitual, por lo cual su adopción de valor pronominal por influencia de nadie debe considerarse sólo una hipótesis). Y aquí el babelismo sigue deparando virtualidades: "La raison est la nature -le natal, le naissant, le naître se poussant vers sa propre inconditionalité. C'est à dire, vers son absoluité: délie de tout, ne pouvant être lien à rien, pouvant n'être que naître" (J.-L. NANCY, Sexistence, cit., p. 77); o no hacer, sino nacer. Et rien de rien: "dans l'anglais du temps de Shakespeare, thing désignait couramment le sexe (l'organe, de l'un et l'autre sexe). Parfois il désignait plutôt le pénis et nothing désignait la vulve (ou l'anus d'un garçon). [.../] Rien donc, la chose minimale, l'être infime et intime dans une imminence, dans une urgence qui se presse. Le réel qui s'impose et qui s'appelle en même temps: qui n'est pas sans s'appeler à être. Qui est dans sa venue, dans sa levée. Le nihilisme a son revers: au verse de la "volonté de volonté» qui veut l'accroissement de sa puissance sur toutes choses, et comme exactement tendu le long d'elle, se trouve le désir levé vers lui-même en tant qu'ouverture, adresse sans destination, salut simplement suspend" (J.-L.. NANCY, Sexistence, cit., p. 61).

56 Cfr. Il Metodo, p. 627.

${ }^{57}$ Cfr. Il Metodo, p. 670. Activar la in-actividad, l'impotenza o la potenza di non essere, que no transita al acto, sino que se retiene, puesto que su acto sería la propia, ¿propia?, potencia. Cfr. G. Agamben, Homo sacer. Il potere sovrano e la nuda vita, Torino: Einaudi 1995, pp. 51-55. O, también: “... notre propre compréhension du mode d'être de la pulsión comme dunamis, ne peut plus s'accommoder de sa lectura metaphysique en termes d'opposition entre puissance et acte. Toute notre intérpretation consiste au contraire à mettre en évidence une dunamis dont la sterèsis interne se traduit par le phénomène de la presence d'une absence. Entre donc en scène, grâce à l'intérpretation heideggérienne des Aristote, cette forme particulière de la temporalité qui se caractericé 
$\mathrm{Y}$, sin embargo, el objeto de estos ejercicios no es un objeto cualquiera ${ }^{58}$. ¿Qué es lo que se presenta o se oculta ahí?, ¿qué se envía o retiene ahí?, ¿qué se siente o no se siente ahí?, ¿qué se distiende o se contrae ahí? El zero es más y menos que un objeto-una cosa, res, o el gallego res, ren: ningunha cousa o moi pouca ${ }^{59}$. Enviar el cero establece una relación hasta donde puede haber relación que no sea relación, por tanto, hasta donde pueda haber relación ( $\mathrm{y}$ no cosa, sustancia, objeto, etc.). Esa relación es la no-relación, pero, también, esa no-relación es la relación ${ }^{60}$.

Por lo pronto, la maestra comparece y se sitúa en el centro, eje de la órbita-pero ¡qué órbita esa del zero!, ¿el vacío?- donde han de revolucionar sus pequeñuelos a su voz, que se profiere con el mismo atractivo con que antes se investía al objeto, con la misma autoridad con que antes se investía al objeto:

Io mi metto in mezzo a loro, che stanno seduti sulle loro seggioline; mi rivolgo ad uno che ha già fatto l'esercizio dei numeri e gli dico: - «Vieni, caro; vieni da me zero volte».

par le mode d'être du pas encore, c'est-à-dire de la suspension et de l'actualisation différée d'une virtualité" (R. Bernet, Force - Pulsion - Désir, cit., p. 20).

58 "... that, jouissance does not exist, and that it is found everywhere [...] for Lacan, that wich exists is a lack. It is from this that he 'derives' the status of enjoyment. The latter is not something that could 'fill up' the lack, because the lack 'is' - that is to say, it is not simply an empty space that might be filled or occupied by anything. We could say that there is a place that is 'ocuppied by the lack' which is 'full of the lack'; and of course, the 'operation' that takes place in this context is that the lack comes to lack (as, for example, in the case of anxiety) ..." (A. Zupančič, Ethics of the Real, London - NY: Verso 2011, p. 242). O, por poner otro ejemplo, en el caso de que me pidan zero baci. Será entonces cuando empiezo a sentir esa falta, ese vacío, esa nada. "Mandami zero baci» no sólo supone que el deseo no alcanza jamás su término; más allá de eso conlleva que esa perpetua insatisfacción pase, o puede pasar, a satisfacerse como tal insatisfacción en su negatividad, devolviendo así al método la eventualidad del acontecimiento que proscribía: "If enjoyment [...] is that which substracts itselffrom the lack [...] we propose the status of enjoyment as that of the 'one-lack-less'. It is that the concept of the drive is aiming at" (id.). "The basic operation of suture is thus that 0 is counted as 1 : the absence of a determination is counted as a positive determination of its own, as in Borges's famous classification of dogs which, includes, as a species, all the dogs not included in the previous species, i.e., the "part of no-part» of the genus dog" (S. ŽIŽEK, Sex and the Failed Absolut, cit., p. 381).

59 La ley del deseo (la pulsión) no resulta de su intrínseca insatisfacción, que compele a que, si me piden dos, quiera dar tres, o mejor cuatro. No se trataría sólo de eso, sino de descubrir que eso no es, That's not It: "If the fundamental constellation of desire implies an infinite and incommensurable measure which makes every given object turn out to be insufficient ('That's not it'), pure desire can be defined as the moment at which desire is forced to say for its own Cause (for its absolute condition): 'That's not It'. This means that the moment of pure desire is, paradoxically, the very moment at which desire loses the foundation of its purity” (A. Zupančič, Ethics of the Real, cit., p. 244. Cfr., también, id. "When Surplus Enjoyment Meets Surplus Value", en J. Clemens and R. GrigG, editors, Jacques Lacan and the Other Side of Psychoanalysis, cit., p. 167, para el "That is not it!» histérico o "the lack as real»). That's not it, that wasn't it, aunque, o porque, once upon a time.

60 'Il s'ensuit que le rapport n'est rien d'étant: rien de distinct, mais la distinction même. Ou plus exactement, c'est le se distinguer en quoi le distinct a sa propriété, et ne l'a que par rapport à d'autres distincts. En se rapportant, le distinct se distingue: c'est-à-dire qu'il se ouvre et se ferme en même temps. Il renvoie à l'autre et il s'en sépare. Le rapport n'est possible qu'en tant qu'il est ce duel [.../] Dire qu'il n'y a pas de rapport, c'est par conséquent énoncer la propriété même du rapport: il doit pour être n'être pas une troisième chose entre les deux [ni uno, ni dos, ni tres...]. Il doit au contraire ouvrir l'entre comme tel il doit ouvrir l'entre-deux par lequel il y a deux. Mais l'entre-deux n'est aucun des deux: il est le vide..." (J.-L. NANCy, L'«il y a» du rapport sexuel, cit., pp. 22 s.). El cero. 
Il bambino quasi sempre corre da me e poi torna al posto: «Ma, figlio mio, tu sei venuto una volta e io ti avvevo detto zero volte». Comincia la meraviglia: «ma allora cosa dovevo fare»? - «Nulla; zero è nulla». "Ma come si fa a far nulla»? - «Non si fa» - «Tu dovevi star fermo; non dovevi muoverti; non dovevi venire nessuna volta: zero volte, niente volte». ${ }^{61}$

('La maravilla!: hacer nada, figlio mio, hacer cero: non, nessuna, niente.)

Todavía se libidiniza más el centro del ejercicio, el lugar de la maestra, ¡el lugar del zero?, en una repetición que no es como aquélla repetición del fatto fondamentale inaugural del método, sino una repetición en la que se despliega toda la gama afectiva del requerimiento de amores. El cortejo, que se vuelve requiebro apasionado para mudarse en exigencia de correspondencia y hasta súplica que desgarra, en un clímax que no se inhibe, sino que parece el clímax de la inhibición, de la no-inhibición, pues-frementi di sapperla obbedire: ¿de la inhibición y/o exhibición? Hasta que el ambiente se vuelve tan tenso que provoca la explosión de la risa y hasta exige un escape, una descarga de esa carga del zero que, por fin, los iguala a todos en su precipitación: venite tutti!

Ripetiamo l'esercizio: "Tu caro, con le tue ditine mandami zero baci»: il bimbo freme, ride e sta fermo. "Hai capito»? ripeto io con voce d'invito quasi appassionata "mandami zero baci! Zero baci!» Fermo. Risa generali. Io faccio la voce grossa come adirandomi delle loro risa e chiamo uno severamente, minacciosamente. «Tu, qui zero volte! Dico... qui subito zero volte; capisci? Dico a te: vieni qui zero volte»! Non si muove. Le risa si fanno più clamorose, eccitate anche dal mutamento del mio contegno, prima di preghiera, poi di minaccia. "Ma insomma» gemo con voce dolente, piangente "perchè non mi baciate, perchè non venite» e tutti gridano ad alta voce mentre gli occhi brillano, quasi lacrimando di gioia e di risa: "zero è nulla! zero è niente»! - «Ah sì»? faccio io sorridendo pacificamente: "ebbene allora venite tutti qui da me una volta»! Essi mi si precipitano intorno. ${ }^{62}$

(;Mándame cero besos!, ¡da cero vueltas!, ¡ven cero veces!, "¿por qué no me besas?, ¿por qué no vienes?».)

${ }_{61}$ Il Metodo, pp. 623s. El cuerpo propio sería expropiado, por obra de esa conminación a "far nulla», de la propiedad o carácter o trazo solipsista que haría de él el punto cero, allí donde se cruzan los ejes de la orientación subjetiva: "Ce que mon corps garde encore de subjectif, quand je fais abstraction de sa fonction comme «organe du vouloir" (et donc aussi de liberté) et même comme «organe de perception", c'est qu'il se comporte comme un centre ou "point zéro d'orientation"” (R. BERnet, Force - Pulsion - Désir, cit., p. 310; a continuación, en francés, la cita husserliana de Idées directrices pour une phénoménologie et une philosophie pures. Livre Troisième: "le corps propre possède alors [...] ce trait distintif, unique en son genre, qu'il porte en soi le point zéro de toutes ces orientations...", en id.). Aquí, en $I l$ Metodo, se trata de otro zero, un cero movilizado por la demanda de envío, un cero en tránsito o, al menos, un conato o ímpetu o impulso de cero en el trance de estar en punto y a punto de-pero también fuera de punto, pasado de punto, desalojado de la subjetividad o, mejor, desalojado como subjetividad. Ese contratiempo divide la puntualidad (espacial) en el mismo punto cero porque el pulso del deseo sufre un síncope y pierde el compás: ni aquí ni allá, ni dentro ni fuera.

62 Id., p. 624. 
La instrucción mandami zero baci, que no es lo mismo que non baciarmi ${ }^{63}$, provoca que la actividad inhibida (menos-que-no-actividad, in-actividad) se conforme en torno a ese vacío (para ello, pues, no valdría uno, ni dos..., pues el uno, o el dos..., me apropiarían como tal propietario o particular, del uno, o del dos... ${ }^{64}$ ). Sin embargo, ¿̇tiene sentido referirse a una conformación?, y si no, menos aún a una conformidad, como si el impulso se hubiera reducido a cero, nada, muerte, varado en nirvana.

¿Quién conforma o confirma la ausencia que deja el yo en su retirada-el vacío $^{65}$, el zero ${ }^{66}$ ? Esa falta excede a cualquier deuda y desborda cualquier intercam-

${ }^{63}$ No es «ino me beses!», orden ante la que me sometería obediente y de la que resultaría mi inmovilidad, y que permitiría imaginar la situación contraria, en la que me dijeran «¡bésame!» pero apartaran de mí los labios, o el pecho, de tal modo que no pudiera besar-como en el juego infantil en que apretando con fuerza mi mano, me dicen «Venga, venga, vete. ¿Por qué no te vas», $<$ Ach geh doch, geh doch, Warum gehst Du nicht?> (F. KafKA, Carta al padre). En esa oposición real de fuerzas o intensidades, en la que los impulsos se reducen y destruyen, substrayéndose entre sí y dando por resultado del juego la privación o el cero. Las intensidades se expresan como distintas (haber y deber, fuerza y debilidad, autoridad y obediencia...), pero reversibles, dando así al trate con la negatividad o tornándola en la indiferencia $($ muerte $=$ nacimiento negativo; nacimiento = muerte negativa (Cfr. I. Kant, Versuch den Begriff der negativen Grössen, 1763). Pero en «Mandemi zero baci» no hay oposición de fuerzas y, por tanto, tampoco reciprocidad o simetría. Es otro zero.

64 "A thing is not reducible to its particular features, it is the One that combines them, and this One is empty, a punctual point of Nothing, and as such again universal: every One is the same One. What is indivisible in a thing is not any of its properties but the Oneness of a thing in distance to properties: Nothing (the void of One-ness) is the heart of a thing, its very self-identity. As Samuel Beckett put it at his Hegelian best: «Everything divides into itself, I suppose». One has to note his precise formulation: it's note that a thing simply divides itself (into two, into its essence and appearance or whatever), it divides into itself, i.e., it no simply divide (distinguish) itself from its environs-it divides itself from itself so that the division that establishes itself is the division into one and nothing" (S. Žıž̌K, Sex and the Failed Absolute, cit., pp. 289 s.).

${ }_{65}$ "Il semble manquer quelque cose: or il ne manque rien. Aucun objet. C'est toujours je qui manque ou qui se manque: de fait, il n'est pas là, il s'absente dans son désir. Car ce n'est pas le sein, il ne lui appartient pas. Il revienta au dehors, c'est au dehors qu'il faut le confier" (J.-L. NANCY, Sexistence, cit., p. 124).

66 "... it is this zero-level tension, the tension that splits from within the Void itself, which engenders the entire movement of the rise of One, Two, etc. [...]" (S. ŽıžEK, Sex and the Failed Absolute, p. 299). El cero contado como uno. Pero cabe preguntarse si esa nada, vacío, cero, que divide al sí mismo en sí mismo, dentro de sí mismo, no lo es en virtud de su ofrecimiento al otro-de la identificación. Y si así fuera, jsería esa la identificación imaginaria del reconocimiento especular en el prójimo, o sería la identificación real de la otreidad insoportable? ("What Levinas obfuscates is the monstrosity of the neighbor, a monstrosity on account of which Lacan applies to the neighbor the term Thing (das Ding), used by Freud to designate the ultimate object of our desires in its unbearable intensity and impenetrability. [...] the neighbor is the (Evil) Thing that potentially lurks beneath every homely human face. [...] What right did he have to stir up her desire? It is from this abyss of the Other as Thing that we can understand what Lacan means by what he calls the 'founding word', statements that confer on a person some symbolic title and make him or her what they are proclaimed to be, constituting their symbolic identity: 'You are my wife, my master...' [...] Lacan's point is that we need this recourse to performativity, to the symbolic engagement, precisely and only in so far as the other whom we confront is not only my mirror-double, someone like me, but also the elusive absolute Other who ultimately remains an unfathomable mystery" (S. ŽıžeK, How to read Lacan, London: Granta Books 2006, pp. 43, 45). Pero ¿y si la privanza de la identidad simbólica conferida desde el abismo de ese gran otro es "you are my zero»?, ¿̨no es esa monstruosidad la monstruosidad del llamamiento del otro dentro del sí mismo que provoca que me dé la vuelta como un guante?: “... ce qui n'est ni chose, ni personne, ni sujet, ni objet, mais entre deux - [/] - l'identification même, et comme l'œil sans vision d'une mêmeté qui se dispose différente, indéfiniment différente - substance non 
bio, por tanto, a cualquier promesa... pero ¿̇no será, en su incondicionalidad - nulla, niente- la condición -y, por lo que comporta su anterioridad, podríamos decir kantianamente la condición trascendental- de la promesa (de amor y muerte)?

Sin embargo, ¿todavía reteniendo una voluntad de goce que requiere la fantasía del goce de la maestra, para así ocupar el sitio del cero, ese círculo encantado que sería el círculo del deseo de la maestra, pero vacío?

Si la ley -la maestra- quiere uno, dos, tres..., puedo imaginar o fantasear o representar, e identificar, e identificarme con ese su deseo, e intercambiarme por ese valor.

Non appena la maestra ha toccato il cuore de' suoi scolari ad uno ad uno, risvegliando e ravvivando in loro la vita com'a un tocco mágico, ella possederà quei cuori, e un segno, una parola saranno sufficienti, perchè ognuno di essi senta la sua presenza, la riconosca e l'ascolti [ed. 1909: ... risvegliando e ravvivando in essi la vita come una fata invisibile - ella possederà tale anime e basterà un cenno, una parola - perchè ciascuno senta lei vivamente, la riconosca e l'ascolti].

Verrà un giorno in cui la maestra, con sua gran meraviglia, si accorgerà che tutti i bambini la obbediscono come miti agnelli lattanti, non solo pronti ad ogni suo segno, ma anche in attesa di esso. Essi la considerano come una persona che dà loro vita e da cui sperano, insaziabili, di ricevere sempre nuova.

L'esperienza ce lo ha rivelato; e ció forma la meraviglia massima di chi visita le «Case dei Bambini»; - la disciplina collettiva si ottiene come per una forza di magia. ${ }^{67}$

(Cada uno, ognuno bambino, un cordero manso y obediente, mito, pero alerta y pronto ante cualquier gesto, un cenno, una (altra) parola, que señale que ella, maestra e mamma, con un toque mágico, desnuda su mammella, don de su vida, che dà loro vita a tutti i bambini, los cuales, tutti, insaziabili, se congregan en el banquete no totémico, identificados en el común de la comunión y, como por arte de magia, forza di magia, resulta la disciplina collettiva. $)^{68}$

substantielle, désir sans manque, tension d'une nature nue vers plus de nudité encore mais vers aucun secret, par aucune transgression. Épanouie ou bien flétrie, jeune ou vieille, gonflée ou flasque, cette nudité est la même, toujours à la conjonction [.../] - parce qu'il faut qu'il y ait disjonction, et séparation, mais non pas cependant parce qu'il y aurait incomplétude, ni manque ni finitude, comme ils disent, mais parce que rien ne vaut seul, tout simplement. [.../]. Porquoi est-ce si difficile à faire entendre? Tellement plus difficile que l'amertume et la mélancolie de l'être-seul, qui ne dit rien d'autre, peut-être?" (J.-L. NANCY, La Naissance des seins, cit., pp. 87s.).

${ }_{67}$ Il Metodo, p. 232, lección de ed. 1950.

68 "L'identification, pour Freud, c'est toujours d'abord l'identification au Père - et le Père veut dire ici: celui qui s'est toujours-déjà identifie, celui qui s'est présenté avant de disparaître, celui qui s'est symbolisé avant d'être symbolique. [/] Or [...], que se passe-t-il dans le repas totémique? [...] ce qui est véritablement dissocié, et 
Siguiendo así aferrado o adherido a esa gran otra, que es la maestra, sin pasar más allá. Pero ¿si quiere zero? Entonces, mi falta (ser-cero, no-tener, casi no-ser) es correlativa de su demanda, y me quedo, en ese trance en que zero vacía y priva-¿cómo oír tal petición sin vaciarme del todo? ${ }^{39}$-, sin pantalla donde reflejar mi fantasía (ser-uno, ser-dos, ser-tres...).

Pero si fuese así, ¿por qué puede, todavía, provocar vergüenza ser-cero?; ¿acaso porque nos ofrecemos a la mirada de esa fata invisibile, y así, ilusionados e ilusos, nos enmarcamos en esa mirada, identificándonos, aun a pesar, o gracias, al zero, que seguiría siendo figura, metáfora, pantalla del deseo, en la que tal vez no se refleja ni esto ni lo otro, pero por ello mismo más poderosa, hasta incomensurable y sublime (suprasensible)?

El emblema con el que sellan mi singularidad, que no puede ser la de ningún otro cualquiera (uno, dos, tres ...), es el cero, nada, nadie, el vacío de la separación, mandami zero baci, que provoca la vergüenza por la fidelidad imposible a tal demanda (amor):

È interesantissimo studiare l'espressione del viso dei possessori [de lo que no se posee:] dello zero: le differenze individuali che ne risultano, sono quasi una rivelazione del «carattere» di ciascuno. Alcuni restano impassibili, con un fare orgoglioso, che tende a nascondere l'interna pena della disillusione; altri manifestano con gesti momentanei l'impressione del disappunto; alcuni non possono nascondere il sorriso che nasce dal sentimento di una situazione singolare, la quale desterà negli altri curiosità; alcuni poi seguono tutti i movimenti dei compagni, fino alla fine dell'esercizio, con evidente espressione mimica di desiderio, quasi d'invidia; altri infine manifestano una subita rassegnazione.

Così è pure interessante la loro espressione nel confessare lo zero, quando si chiede, durante la verifica: «e tu, non hai preso nulla»? - «Ho lo zero» - «è zero» - «avevo zero». Queste sono le risposte uniformi del linguaggio parlato, ma la mimica espressiva, il tono della voce, esprimono sentimenti ben diversi. Rari sono quelli che con fare ardito, sembrano concedere la spiegazione a un fatto straordinario: i più sono crucciati, o rassegnati.

Bisogna perciò dare delle lezioni sul contegno: — «badate, è difficile tenerlo il segreto dello zero - lo zero sfugge dal naso: fate i disinvolti, non lasciate capire che non avete nulla». Infatti dopo qualche tempo, l'orgoglio della dignità ha il sopravvento e i piccini si abituano a ricevere

incorporé, c'est une nourriture qui répète la commune substance maternelle. [...] l'identification du clan, dans son moment le plus "régressif», le plus «intérieur», dans le moment de l'assimilation qui fait des semblables - et qui les disocie - est identification à la Mère" (Ph. Lacoue-Labarthe et J.- L. Nancy, La panique politique suivi de Le peuple juif ne rêve pas, Christian Bourgois éditeur 2013, pp. 73 s.).

69 "The call is not necessarily of language or entirely without language. It is hard to situate with any certainty. In Sein und Zeit, Heidegger had said of the call-the aphonic call of conscience-that it comes within me and from beyond me. In order to heed the call one has to have emptied onself, have undergone a personal kenosis (which, of the extent that it empties, drops off the "personal»)" (A. Ronell, Loser Sons, cit., p. 166). 
lo zero e i numeri piccoli, con disinvoltura, contenti di non manifestare più i piccoli sentimenti dei quali prima erano schiavi ${ }^{70}$.

("Fati i disinvolti, non lasciate capire de che non avete nulla». "Haz como si nada pasara, sin dar a entender que no tienes nada". Con el tiempo, el orgullo, o el honor, prevalece, orgullo de ser marcado como poca cosa, como nada o casi nada: "... si abituano a ricevere lo zero e $i$ numeri piccoli».)

Sería ese el carácter del deseo (¿masculino?), en el que, aunque se caigan el uno, el dos, el tres... y sintamos la nada del cero, hasta ese cero pudiera hacer de pantalla a la fantasía del deseo de la maestra e mamma, para estar a la altura del deseo de la maestra y reflejar el goce perverso de mi sujeción a ese significante otorgado ${ }^{71}$.

¿Sería posible atravesar ese rasgo?

Sería ese el carácter del deseo (¿femenino? ${ }^{72}$, pues, más que vergüenza, confusión. Confusión que provoca el enigma del deseo, il segreto dello zero, su falta de objeto, su ningún objeto, su infinitud que no es, o al menos no sólo, la de su insatisfacción, sino la de su inapropiabilidad: "[il bambino] se in sorte gli toccò lo zero, resta pazientemente a mani vuote". ${ }^{73}$

¿No es, entonces, su imposibilidad de ser el secreto de su inapropiabilidad, su no-ser en propio, propiamente?: badate, è difficile tenerlo il segreto dello zero - lo zero sfugge dal naso ${ }^{74}$ — como el amor y la muerte.

${ }^{70}$ Id., p. 628. "The term [honor] forms a pair with the word shame, shame hidden by modesty, but heightened, enlarged, by honor". Y, más adelante: "Lacan, not yet having formalized this master signifier, calls it, in his text on Gide, the subject's "emblem", a term intended to resonate with honor: "The emblem that the iron of an encounter has imprimed upon the subject» He also says, "The seal is not only an imprint but a hieroglyph as well» $[/ \ldots]$. One can anticipate that this unusual mark is what he will later call the master signifier that marks the subject with an ineffaceable singularity" (J.-A. Miller, "On shame”, en J. Clemens, R. Grigg (Eds.), Jacques Lacan and the Other Side of Psychoanalysis. Durham and London: Duke University Press 2006, p. 18 y p. 20). Asimismo, si bien referido a la posición del padre, "shame, because he must represent a master signifier in the full consciousness that this is impossible [...]. Shame, too, because he will never be able to fill in the lack, because he can only pass it on to the next generation -which is, incidentally, also an expresion of love: giving what you don't have ("L'amour, c'est donner ce qu'on n'a pas»)" (P. VerhaEghe, "Enjoyment and Impossibility", id., p. 47; cfr., también, D. Hoens, "Toward a New Perversión: Psychoanalysis", id., pp. 94-98). Donner ce quion n'a pas: zero baci.

71 Cfr. J.-A. Miller, “Des semblants entre les sexes”: La cause freudienne 36, 1997, pp. 14 y s. "Il y a une prégnance très spéciale du fantasme dans la sexuation de l'homme, et parfois, loin que le fantasme permette une traversée du côté du désir masculin, on observe, au contraire, comme une compression de celui-ci” (p. 15).

72 “... qu'au momment où un homme rencontré, la fonction $\Phi$ [el falo] se fait plus insistente, alors que lorsque se déploient les voies de son désir pour une femme, il a chance d'accéder à A, c'est-à-dire à savoir que l'Autre n'existe pas" (J.-A. Miller, "Des semblants dans la relation entre les sexes", cit., p. 14).

73 Il Metodo, p. 670. "... when we pass from perversion to the analytic social link, the agent (analyst) reduces himself to the void [para nosotros, veremos, "el deseo del cero emplazado en el sitio de la maestra»], which provoques the subject confronting the truth of his desire" (S. ŽIžEK, "Object a in Social Links", en J. Clemens AND R. Grigg, editors, Jacques Lacan and the Other Side of Psychoanalysis, cit., p. 115.

${ }^{74} \mathrm{Su}$ no-ser, su imposibilidad, pero no su irrealidad. "I will leave the last word to Beckett. [...] he said in a letter in 1967: "If I were in the unenviable position of having to study my work, my point of departure would be the «Naught is more real...». [...] in Murphy (1938), his early novel, and then again in Malone dies (1951), but 
Ni uno, ni dos, ni tres, ni... La mala infinitud del objeto del deseo que gira en torno a su carencia, carencia que me identifica, al distinguirme en relación a esa otra, en la separación de esa otra que me pide cero, a mí y no a otro, porque le falta cero, a ella también, o, más bien, tampoco ${ }^{75}$.

La relación de la privación, la reciprocidad de la privación, relación y reciprocidad en la que no hay activo ni pasivo según los que diferenciarse o identificarse, pues ni siquiera hay identidad o mismidad en que o según la que diferenciarse $y$, por tanto, no ha lugar para la reciprocidad.

Pero ¿no se abre otra diferencia y otra identidad, según otra identificación?

Zero: el lugar y no-lugar donde el lenguaje se hace coextensivo con el corte (del discurso).

En esa grieta que abre la comanda en sordina: «mandami zero baci» ${ }^{76}$ se precipitan sujeto y objeto, como si no fueran sino una ilusión («érase una vez»).

La ilusión de la pulsión.

Porque es tan poco lo que nos identifica, el zero, y, sin embargo, en esa relación sin objeto, o casi - 'casisinòbjeto', 'sincàsiobjeto' ${ }^{77}$-, la relación es así sorprendida.

Sin duda mandami zero baci es la comanda en la cual me suspendo, avergonzado, no con una vergüenza cualquiera, sino con la vergüenza que corresponde a la máxima falta y debilidad: la del sujeto-su cero. No poder corresponder, o corresponder en el cero. Pero, también, se trata de la confusión del amor, si el amor es dar lo que

more emphatically, $\delta \dot{\varepsilon} \nu$ as "not nothing» is precisely the "unnullable least» that Beckett singled out in one of his last works, Worstward Ho! (1981)" (M. Dolar, “One Split into Two", cit., p. 91. No obstante, en ese artículo no se piensa el cero, sino en "the figure of the two which resists the One and defies counting", id.).

75 "Lorsque je baise, donc, je suis baisé, mais comment l'entendre? Qui baise qui, et que veut dire baiser et/ou l'être (baisé)?” (J.-L. NANCY, L'«ily a» du rapport sexuel, cit., p. 14; la traducción inglesa: "when I fuck, I'm fucked, but how are we to understand this. Who fucks whom, and what does it mean to fuck or to be fucked?", Corpus II. Writings on Sexuality. Translated by Anne O’Byrne, New York: Fordham University Press 2013, p. 3). Cuando yo doy cero besos, me dan cero besos, pero ¿cómo entenderlo? ¿Quién besa a quién, y qué quiere decir besar y/o serlo (besado) cero veces, cada vez cero veces, cero besos? A la respuesta apunta la barra: «et/ou», cuyos términos "et» 'y' "ou» son también «et» 'o' "ou», barra que barra la identificación (inclusión) en la repetición (exclusión): y..., o...; en la diferencia que (no) es nada de otro, "rien d'autre: l'autre même, l'autre au sein du même comme son désir même. Le naître en tant que nêtre rien d'autre que sa propre altération et le jouir comme l'épreuve d'être ainsi altéré dans son être" (J.-L. NANCY, Sexistence, p. 79). Vida y/o muerte.

76 "Être défait par un autre est une nécessité primaire, une angoisse à coup sûr, mais aussi une chance - d'être interpellé, reclamé, lié à ce qui n'est pas moi, et aussi d'être ému, d'être obligé d'agir, de m'adresser ailleurs, et de ne plus faire ainsi du «je» autonome une sorte de possession” (J. Butler, Le Récit de soi, trad. B. Ambroise et V. Aucouturier, Paris: PUF 2007, p. 137; cit. en G. Didi-Huberman, Désire Désobéir. Ce qui nous soulève, 1, Paris: Les Éditions de Minuit 2019, p. 492). Pero, aquí, nada de ser movido, d'être ému, nada de ser obligado a intervenir, d'être obligé d'agir, pero sí dirigirme a otra parte, ailleurs, más allá, fuera-de-mí.

$77 \mathrm{Ni}$ siquiera el eco para la ecolalia del decirse o para la ecopraxia del besarse, si acaso, el eco del silencio que precede al decir y el eco de la privación que precede al hacer; entonces, el eco de la receptividad que precede al mandamiento: la receptividad, la vulnerabilidad. 
no se tiene ${ }^{78}$ ¿ ¿también darse lo que no se tiene? Dar lo que no se tiene (zero) conlleva presentar mi, ¿̇mi?, impotencia más originaria y, por consiguiente, la demanda que me antecede, anacrónicamente, abriendo, mandami..., y cerrando, ... zero, el entredós de la (no-)relación-baciarsi.

¿Qué sentido tiene esa demanda o comanda que exige el envío de zero baci?, ¿qué sentido habría de tener sino el del envío, no de esto o lo otro (de este uno, o de este dos...), sino zero, el del envío mismo, y no lo enviado, envío cuya insustancialidad abre -destina- y cierra -interrumpe- para que el agujero de la inhibición se exhiba como un entredós? ${ }^{79}$

$$
Y / o, o / y, y / o \ldots
$$

\footnotetext{
78 “Qu'il y ait amour de la faiblesse, sans doute est-ce là l'essence de l'amour. Comme je l'ai dit, l'amour, c'est donner ce qu'on n'pas, à savoir ce qui pourrait réparer cette faiblesse originelle” (J. LacAn, Le Séminaire. Libro XVII. L'envers de la psychoanalyse. (1969-1970), Paris: Éditions du Seuil, Mars 1991, p. 57). Sería ese el amor del analista, el amor de la verdad. "Referring to Seminar VIII, one could say that the analyst, in order to become an analyst, has left the place of the eromenos (beloved) for the place of the erastes (lover). This shift is what Lacan calls "the miracle of love.» It means that one is desired as an object and is capable of desiring back from this object position. "To love» means to desire the other as the subject of a desire. [...]. The analyst loves the analysand not for this or that personal trait (love de dicto) and not because of some essential humanity (love de re), but for an inhuman [singular, extrasymbolic] desire. This is how Lacan understand Freud's quote: love of truth is love of the essential weakness human beings are marked by. "What is the love of truth" he asks. "The love of truth is the love of this weakness whose veil we have lifted, it's the love of what truth hides, which is called castration... That there is a love of weakness is no doubt the essence of love»" (D. Hoens, "Toward a New Perversion: Psychoanalysis", cit., p. 95; y más adelante: "If we add [...], in this position [of the analyst], of taking the appearance of the subject as an isolated signifier (S / S1) [el cero que representamos, que nos hace absolutamente singulares], that is, as a sign, one could think of a specific intersubjetive relation we can love”, p. 98). Pero ise da o se pide un objeto?, ¿un sujeto?, ¿o, más bien, ocurre que la debilidad de la verdad y la verdad de la debilidad es que no tienen objeto ni son del sujeto, sino anteriores, como un anacronismo para sujetos y objetos?

79 "Le sens se dit pour se faire et se fait pour se dire. Il ne se tient pas seulement dans l'envoi des existants les uns aux autres: cet envoi -cet envol, cette levée- s'expédie lui même toujours hors de soi. Pas plus qu'il n'y a "l'être» il n'y a l' "avec». C'est bien là que se joue la formidable ambivalence et ambiguïté de tout ce qui relève du com: communication, commerce, copule (qui est fait de co et de apio, lier). L' "avec» n'est jamais une chose, une substance ni un sujet. Il est l'elément du seul sens, dans tous ses sens, c'est-à-dire de toutes les façons du sentir, recevoir ou repousser un dehors, n'être jamais «dedans» sans ce dehors qui viene et qui s'écarte” (J.-L. NANCY, Sexistence, cit., p. 51).
} 


\section{REFERENCIAS Bibliográficas}

Agamben, G. (1995), Homo sacer. Il potere sovrano e la nuda vita, Torino: Einaudi.

BaAs, B, "Ulysse en Baltique": Filozofski vestnik, volume XXXV, number 2, 2014, pp. 11-32.

Bernet, R. (2013), Force - Pulsion - Désir. Une autre philosophie de la psychanalyse, Paris: Libraire Philosophique J. Vrin.

Clemens, J., Grigg, R. (editors), (2006), Jacques Lacan and the Other Side of Psychoanalysis: Reflections on Seminar XVII.

Didi-Huberman, G. (2019), Désire Désobéir. Ce qui nous soulève, 1, Paris: Les Éditions de Minuit.

Dolar, M., "One Splits into Two": Das Magazin des Instituts für Theorie, 31, No 14/15, pp. 94-99.

Foucault, M. (1997), Il faut défendre la société. Cours au Collège de France, 1976, Paris: Gallimard - Seuil.

Deleuze, G., Guattari, F. (2004), Mil mesetas. Capitalismo y esquizofrenia, traducción de José Vázquez Pérez con la colaboración de Umbelina Larraceleta, Valencia: Pre-Textos.

LaCan, J. (1987), El Seminario. Libro 11. Los cuatro conceptos fundamentales del psicoanálisis. 1964. Texto establecido por Jacques-Alain Miller. Traducción de Juan Luis Delmont-Mauri y Julieta Sucre. Buenos Aires - Barcelona - México: Ediciones Paidós.

Lacan, J. (1991), Le Séminaire. Libro XVII. L'envers de la psychoanalyse. (19691970), Paris: Éditions du Seuil.

LaCan, J. (2010), El Seminario. Libro 10. La Angustia. 1962-1963. Texto establecido por Jacques-Alain Miller. Traducción de Enric Berenguer, corrección de Gabriela Ubaldini. Buenos Aires - Barcelona - México: Editorial Paidós.

Montessori, M. (2000) Il Metodo della Pedagogía Scientifica applicato all'educazione infantile nelle Case dei Bambini. Edizione critica, Roma: Edizioni Nazionale Montessori.

Montessori, M. (2000), L'autoeducazione nelle scuole elementari, Milano: Garzanti. 
NAncy, J.-L. (2001) L'«il y a» du rapport sexuel, Paris: Éditions Galilée.

Nancy, J.-L. (2013), Corpus II. Writings on Sexuality. Translated by Anne O’Byrne, New York: Fordham University Press.

Nancy, J.-L. (2017), Sexistence. Accompagné d'un frontispice de Miquel Barceló, Paris: Éditions Galilée.

P. Lacou-Labarthe, Ph,Nancy, J.-L. (2013), La panique politique suivi de Le peuple juifne rêve pas, Christian Bourgois éditeur.

Ronell, A. (2012), Loser Sons. Politics and Authority, Urbana, Chicago and Springfield: University of Illinois Press.

VÁzquez-Romero, J. M. (2018), "Dialécticas idiotas (entre salvajes y zombis)": Pensamiento, vol. 74, pp. 853-882.

ŽıžeK, S. (2006), How to read Lacan, London: Granta Books.

ŽıžEK, S. (2020), Sex and the Failed Absolute, London - New York: Bloomsbury Academic.

Zupančič, A. (2011), Ethics of the Real, London - NY: Verso.

Zupančič, A. (2017), What is Sex, Cambridge, Mass. - London: The MIT Press.

DOI: https://doi.org/10.15366/bp.2020.24.025

Bajo Palabra. II Época. No 24. Pgs: 495-524 